\title{
Novel antibiotic screening methods to awaken silent or cryptic secondary metabolic pathways in actinomycetes
}

\author{
Hiroyasu Onaka
}

Streptomyces have the potential to produce more than $\mathbf{3 0}$ secondary metabolites; however, the expression of most metabolite biosynthetic gene clusters is cryptic or silent. Indeed, the expression of these genes is conditional and dependent on culture conditions. To activate such gene clusters, many methods have been developed. In this review, I describe two activation approaches developed in my laboratory: use of the activation mediator goadsporin and combined-culture. Goadsporin is a chemical substance isolated from Streptomyces sp. TP-A0584; it induces secondary metabolism and sporulation of many Streptomyces species. Combined-culture is a co-culture method to activate secondary metabolism in Streptomyces.

The activator strains are mycolic acid-containing bacteria and $~ 90 \%$ of Streptomyces species show changes in secondary metabolism in combined-culture compared with pure culture. Thus, both methods may have applications in natural product-based drug discovery.

The Journal of Antibiotics (2017) 70, 865-870; doi:10.1038/ja.2017.51; published online 26 April 2017

\section{INTRODUCTION}

Natural products, in particular secondary metabolites derived from actinomycetes, such as streptomycin, have contributed substantially to modern medical care. However, most pharmaceutical companies have recently discontinued natural product screening, and natural product-based innovative drug development is losing momentum, primarily because the rediscovery of known compounds often occurs through bioassay-guided screening. Thus, natural product screening seems to be less efficient than screening of synthetic compound libraries. Moreover, even if new compounds are found from natural sources, there are often problems with productivity or stability of the identified compounds. Pharmaceutical companies typically own enormous libraries consisting of millions of synthetic compounds and are prioritizing high-throughput screening assays using these synthetic compound libraries. However, natural products are still an attractive and indispensable resource, because the core skeletons of most natural products, such as the antiparasitic drug Ivermectin, the antihyperlipidemic drug Pravastatin and the anticancer agent Eribulin, have unique chemical structures only found in nature and not conceived by humans. ${ }^{1}$ Accordingly, screening of natural products is important to identify novel medicinal candidates with a variety of chemical structures.

Since the early 2000s, novel methods of genome analysis have been developed, facilitating major advancements in our knowledge of natural product biosynthesis in many organisms. For example, genome analysis in Streptomyces spp. has revealed that these organisms contain over 30 secondary metabolite biosynthetic gene clusters in the genome, suggesting that Streptomyces has the potential to produce over 30 secondary metabolites in each strain. ${ }^{2}$ However, only a few metabolites are detected in culture broth under laboratory conditions because of conditional or low production of these metabolites. Thus, novel techniques are needed to access the biosynthetic potential of these compounds, including approaches involving modification of nutrient conditions in medium, cocultivation with other microorganisms or chemical elicitors, and the genetic recombination of biosynthetic gene clusters. ${ }^{3-5}$

In this review, I describe two methods for activation of secondary metabolism, that is, combined culture and use of goadsporin.

\section{GOADSPORIN: SECONDARY METABOLISM ACTIVATION BY A CHEMICAL SUBSTANCE}

Isolation of goadsporin

Autoregulators ( $c f . \mathrm{A}$-factor ${ }^{6}$ ) are hormone-like compounds that are widely distributed in Streptomyces species and are known to induce secondary metabolism. However, no compounds inducing secondary metabolism in other Streptomyces have been isolated to date. We identified an autoregulator-like compound that activates secondary metabolism not only in the producing strain but also in a wide variety of Streptomyces species. To screen the compound, we used Streptomyces lividans, which is a conditional producer of two red pigments, actinorhodin and undecylprodigiosin, as the indicator strain in the screening assay. The production of these pigments is

Department of Biotechnology, Graduate School of Agricultural and Life Sciences, The University of Tokyo, Tokyo, Japan

Correspondence: Professor H Onaka, Department of Biotechnology, Graduate School of Agricultural and Life Sciences, The University of Tokyo, 1-1-1 Yayoi, Bunkyo-ku, Tokyo 113-8657, Japan.

E-mail: aonaka@mail.ecc.u-tokyo.ac.jp

Hiroyasu Onaka was awarded the Sumiki-Umezawa Memorial Award from the Japan Antibiotics Research Association in 2016 . This review article is based on his award-winning research.

Received 12 January 2017; revised 23 March 2017; accepted 24 March 2017; published online 26 April 2017 
a

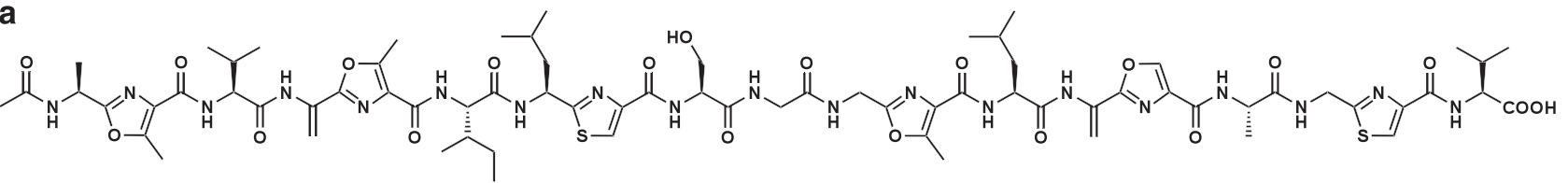

b

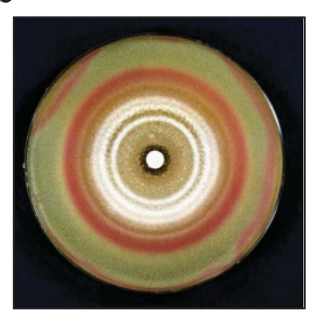

Streptomyces lividans

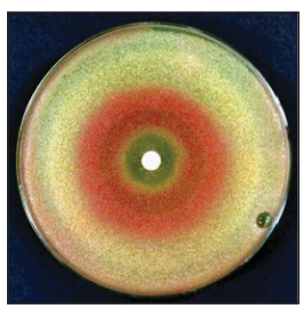

Streptomyces coelicolor A3(2) d

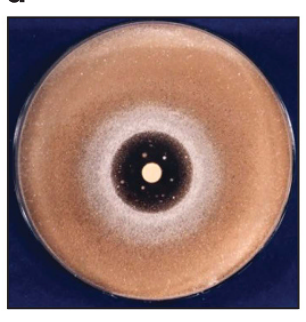

Streptomyces avermitilis e

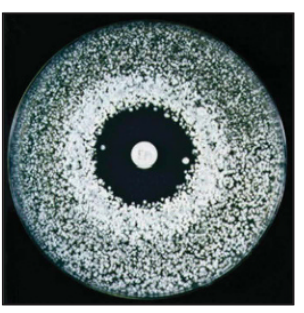

Micromonospora sp. TP-A0184 f

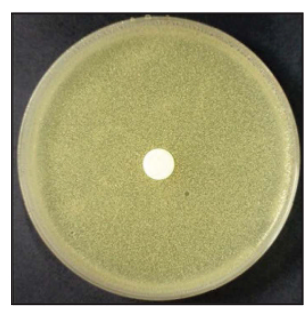

Streptomyces sp. TP-A0584

Figure 1 Chemical structure and biological activity of goadsporin. (a) Chemical structure of goadsporin. (b-f) Biological activity of goadsporin. Five micrograms of goadsporin was absorbed in the paper discs, and the discs were placed on the center of solid plates on which Streptomyces strains were overlaid. (b) S. lividans TK23, (c) Streptomyces coelicolor A3(2), (d) Streptomyces avermitilis, (e) Micromonospora sp. TP-A0184 and (f) Streptomyces sp. TP-A0584, the goadsporin producer.

conditional, and S. lividans does not produce these pigments under normal laboratory growth conditions. The spore suspension of S. lividans was overlaid on Bennett's glucose agar plates on which the red pigments are not produced, and paper discs absorbed with butanol extracts of bacterial broth were placed on the plates. The bacterial broth was prepared from 400 actinomycetes isolated from soil samples and red pigment production around the paper disks was observed. After 3 days of culture, one active compound was found in the culture broth of Streptomyces sp. TP-A0584. ${ }^{7}$ This active compound, designated goadsporin, is a peptide compound containing azole rings and a dehydroalanine structure (Figure 1a). ${ }^{8}$ Goadsporin can induce red pigment production by S. lividans and can promote sporulation; however, when used at more than $1 \mu \mathrm{M}$, goadsporin inhibits the growth of $S$. lividans (Figure 1b). In addition, sporulation and growth inhibition were observed in $\sim 80 \%$ of examined actinomycetes ( 32 out of 42 strains; Figures 1c-e). In contrast, no changes were observed when goadsporin was added to its own producer, Streptomyces sp. TP-A0584 (Figure 1f). This result suggested that Streptomyces sp. TP-A0584 showed self-resistance against goadsporin.

\section{The goadsporin biosynthetic gene cluster}

Based on the amino acid sequence of the goadsporin peptide, we generated a synthetic DNA probe and the goadsporin biosynthesis gene cluster was cloned by Southern hybridization and colony hybridization. The goadsporin biosynthetic gene cluster contains 10 genes spanning $20 \mathrm{~kb}$ on the genome. The godA gene, encoding 49 residues, produces the structure peptide of goadsporin (Figure 2a). Five genes, godD, godE, godF, godG and godH, encode enzymes involved in posttranslational modifications. ${ }^{9}$ In the goadsporin biosynthesis pathway, the precursor peptide of goadsporin is translated by the ribosome machinery; the formation of azole rings is subsequently catalyzed by GodD and GodE, and the formation of dehydroalanine is catalyzed by GodF and GodG. ${ }^{10}$ Finally, the leader peptide of 30 residues attaching at the N-terminal end of GodA is digested by an endogenous peptidase and subsequent N-terminal acetylation by GodH gives rise to goadsporin. Recently, a one-pot synthesis method for goadsporin using enzymes reconstituted in an in vitro translation system was achieved and a rapid analog construction system was established. ${ }^{11}$ In addition to the biosynthetic enzyme genes, the goadsporin biosynthetic gene cluster contains the $\mathrm{ABC}$ transporters $\operatorname{godB}$ and $\operatorname{god} C$, a transcriptional regulator $\operatorname{god} R,{ }^{12}$ and a self-resistance gene godI.

The goadsporin self-resistance gene, godI

GodI is highly homologous to Ffh, which is an essential component of the signal recognition particle (SRP). The SRP consists of Ffh and 4.5S RNA. Importantly, the SRP recognizes the N-terminal signal peptide of proteins translated by ribosomes and connects with this peptide. The SRP then localizes to an SRP receptor existing on the cell membrane. Thus, proteins containing the signal peptide are folded near the cell membrane, and membrane proteins and extracellular proteins can be localized. Our genetic experiments revealed that the ffh homolog godI conferred goadsporin-resistance in the producing strain. S. lividans harboring godI grew on medium containing $30 \mu \mathrm{g} \mathrm{ml}^{-1}$ goadsporin (Figure $2 \mathrm{~b}$ ). In contrast, although an additional ffh existed in the TP-A0584 genome, the godI disruptant did not grow in medium containing $7 \mu \mathrm{g} \mathrm{ml}^{-1}$ goadsporin (Figure 2c). ${ }^{9}$

\section{Proposed mechanism of growth inhibition and secondary} metabolism induction by goadsporin

From our results, we proposed the following mechanism of growth inhibition and secondary metabolism induction by goadsporin. The direct target of goadsporin is Ffh. When goadsporin is added to the culture, Ffh, which binds directly to goadsporin, cannot function and secreted or membrane proteins are not translocated normally, leading to growth inhibition. In contrast, GodI, which is a goadsporinresistant Ffh, cannot bind to goadsporin and is substituted for Ffh. At sublethal concentrations, induction of sporulation and secondary metabolism are observed in almost Streptomyces species. Thus, some stresses result in incomplete protein translocation and facilitate physiological changes in the cell.

Notably, no studies have yet described the relationships between protein translocation and secondary metabolism/cell differentiation in actinomycetes; thus, we expect that the mode of action of goadsporin may lead to the discovery of novel regulatory systems for secondary metabolism. In addition, these findings may also facilitate 
a

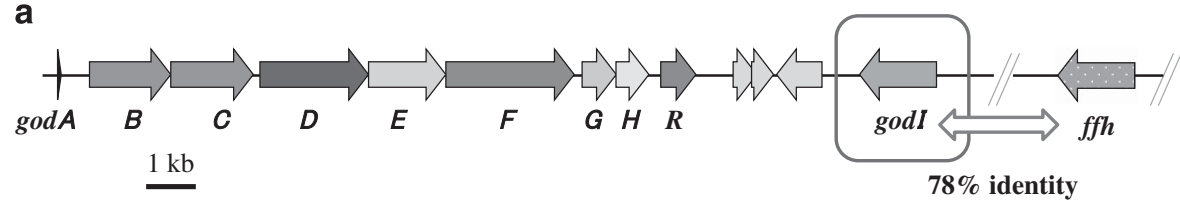

b

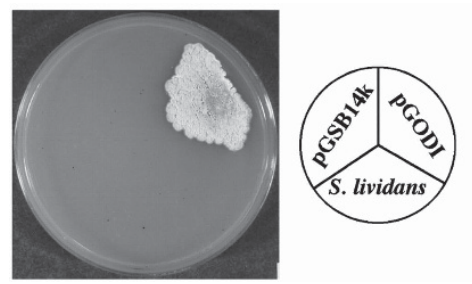

c

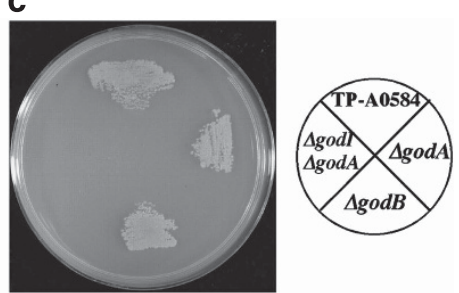

Figure 2 Goadsporin biosynthetic gene cluster and its resistance. (a) Gene organization of the goadsporin biosynthetic gene cluster in Streptomyces sp. TP-A0584. The Ffh gene is located apart from the god gene cluster in the TP-0584 genome. (b) S. lividans harboring godl grew on medium containing $30 \mu \mathrm{g} \mathrm{ml}^{-1}$ goadsporin. (c) The godl disruptant did not grow in medium containing $7 \mu \mathrm{g} \mathrm{ml}{ }^{-1}$ goadsporin. A full colour version of this figure is available at the Journal of Antibiotics journal online.

the development of novel antibiotics through knowledge of the antimicrobial mechanism of goadsporin.

\section{COMBINED-CULTURE: SECONDARY METABOLISM ACTIVATION BY PHYSICAL CONTACT}

Discovery of Tsukamurella pulmonis, an activator of secondary metabolism in actinomycetes

Streptomyces species are mainly found in the soil environment. Soil is a habitat containing many types of microorganisms and these microorganisms may compete with each other to survive in the environment. However, it is not clear why microorganisms produce secondary metabolites, such as antibiotics. Secondary metabolism is not essential under pure culture conditions in the laboratory; however, in the soil environment, secondary metabolism may be essential. If actinomycetes that inhabit the soil environment are influenced by other microbes, secondary metabolism in actinomycetes may not only control the other microbes but also be controlled by them. Based on this hypothesis, we then began to isolate bacteria that activated secondary metabolism in actinomycetes. We used S. lividans as an indicator for secondary metabolism activation, similar to the method used for goadsporin discovery. S. lividans has the potential to produce two red pigments, actinorhodin and undecylprodigiosin. We screened for microorganisms that guide red pigment production in cultures on Bennett's glucose medium. When strains isolated from soil samples were spotted on medium overlaid by $S$. lividans, one microorganism, TP-B0596, was identified (Figures 3a and b). ${ }^{13}$ 16S rDNA analysis identified the TP-B0596 strain as Tsukamurella pulmonis. Furthermore, red pigment-inducing activity in S. lividans was examined for species closely related to $T$. pulmonis, and 3 out of 4 strains examined showed similar red pigment-inducing activity (Figure 3c). Similar activities were also observed in seven genera, including Dietzia, Rhodococcus, Nocardia, Williamsia, Gordonia, Mycobacterium and Corynebacterium, which are closely related to the Tsukamurella genus. Notably, all of these strains have a mycolic acid layer on the outside of the cells (mycolic acid-containing bacteria (MACB)). However, when only mycolic acid was extracted from these strains and added into the culture broth of $S$. lividans, no red pigment-inducing activity was observed.
Red pigment production by S. lividans is induced by physical contact with MACB

Analysis of induction conditions by T. pulmonis revealed that red pigment production by $S$. lividans is induced by physical contact with MACB. The liquid culture of T. pulmonis extracted with $n$-butanol or sterilized using a $0.2 \mu \mathrm{m}$ filter did not induce any pigment production. Moreover, liquid culture broth sterilized in an autoclave at $121^{\circ} \mathrm{C}$ for $30 \mathrm{~min}$ also did not show this inducing ability. In liquid culture with a dialysis flask, which consisted of two compartments partitioned with a $0.2 \mu \mathrm{m}$ membrane filter, T. pulmonis and S. lividans were independently cultured in each compartment over 7 days, whereas S. lividans did not produce any red pigments (Figure 4a). ${ }^{13}$ In addition, in the solid plate culture, red pigment production was induced only at the cell-to-cell interaction site of both strains (Figure $4 \mathrm{~b}$ ). These results strongly suggested that pigment induction was mediated by cell-to-cell physical contact and not by chemical substances, such as A-factor-like autoregulators or goadsporin.

\section{Secondary metabolite screening using combined-culture}

Secondary metabolism induction by MACB occurs not only in $S$. lividans but also in other Streptomyces spp. The secondary metabolite profiles of the combined-cultures and pure cultures were compared. A total of 112 strains of actinomycetes isolated from soil samples collected in the Hokuriku district of Japan were subjected to combined-culture with T. pulmonis. After 7 days of cultivation, the culture broths were extracted with n-butanol and subjected to HPLC analysis. The elution profiles of secondary metabolites were monitored at $254 \mathrm{~nm}$. The corresponding HPLC peaks were compared between the pure cultures and combined-cultures, and the results showed that 99 of 112 strains exhibited changes in secondary metabolism patterns in the combined culture. ${ }^{13}$ Among these strains, 41 produced new metabolites only in the combined-culture, 87 strains showed increased or decreased amounts of metabolites and 12 strains showed no significant changes in combined-culture. These results revealed that $T$. pulmonis altered the secondary metabolites of actinomycetes with a high efficiency of $88 \%$ (99/112). We then applied the combined-culture method to antibiotic screening. As a result, we found that Streptomyces endus S522 produced antibiotics only in combined-culture. These antibacterial substances were then isolated and the chemical structures were determined. ${ }^{13}$ Two types of 
a

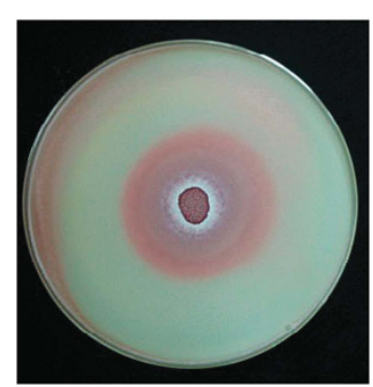

b

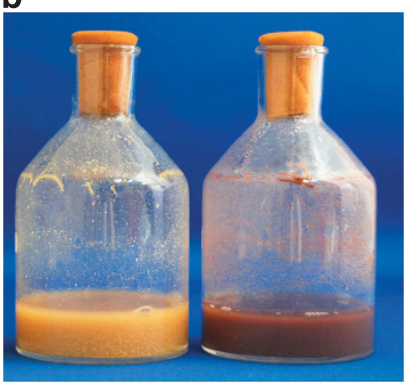

C

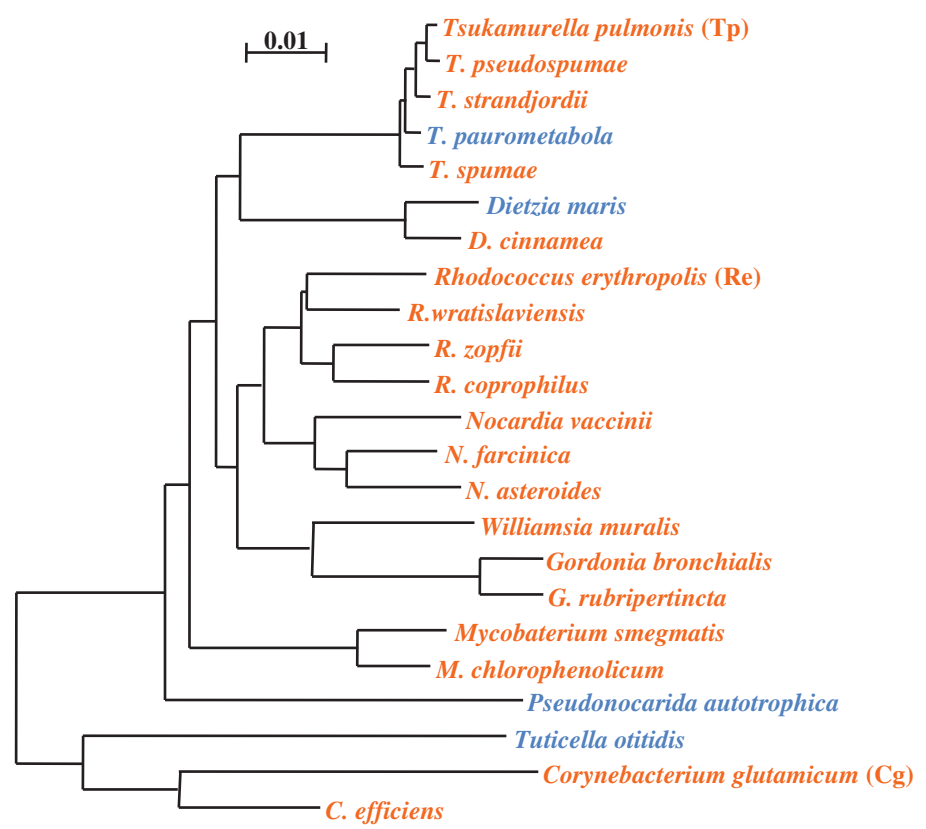

Figure 3 Induction of red pigment production by combined culture and the phylogenetic tree of MACB. (a) T. pulmonis TP-B0596 shows red pigment induction activity. TP-B0596 was spotted on the center of Bennett's glucose plate on which Streptomyces strains were overlaid. (b) Liquid culture of combined-culture with S. lividans and T. pulmonis. The medium was A-3M. (c) Phylogenetic tree of MACB. Red colored strains produced red pigment.

a

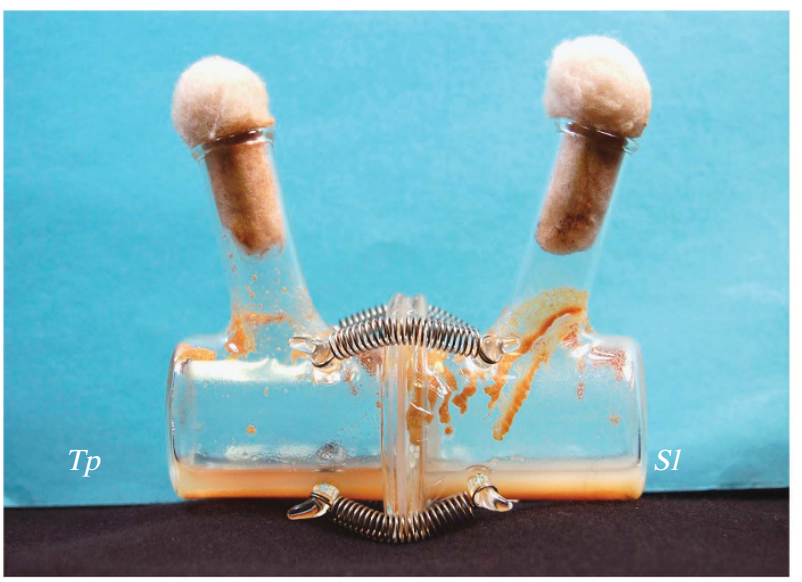

b

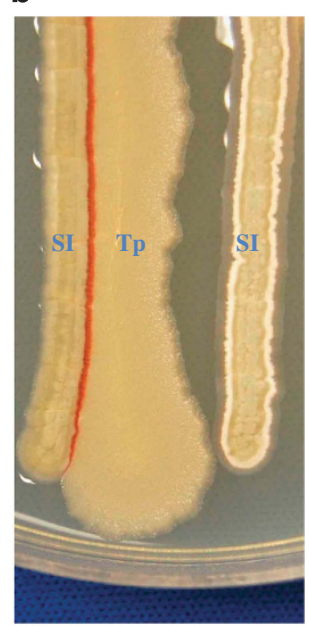

Figure 4 Cell-to-cell interaction is one of important factor in combined-culture. (a) Liquid culture with a dialysis flask partitioned with a $0.2 \mu \mathrm{m}$ membrane filter. T. pulmonis (left) and S. lividans (right) were independently cultured in each compartment over 7 days. (b) Cell-to-cell interactions on the Bennett's glucose agar medium. SI, S. lividans, Tp, T. pulmonis.

new polyketide compounds exhibiting novel heterocyclic ring structures (Figure 5), designated alchivemycin A and B, were identified.

Alchivemycin A (1) exhibits antimicrobial activity when used at an extremely low concentration of $0.06 \mu \mathrm{g} \mathrm{ml}^{-1}$ against Micrococcus luteus and inhibits the invasion of murine colon carcinoma 26-L5 cells into Matrigel with a $\mathrm{IC}_{50}$ of $0.34 \mu \mathrm{M}$ without showing cytotoxic effects. This compounds also lacked significant cytotoxity against murine renal carcinoma Renca cells and human umbilical vein endothelial cells. ${ }^{14,15}$ Furthermore, by screening for novel secondary metabolites using combined-culture (Figure 5), Arcyriaflavin E (2), which has an indolocarbazole skeleton, was identified from the combined-culture broth of Streptomyces cinnamoneus NBRC 13823 and T. pulmonis. Arcyriaflavin E shows cytotoxicity in human cells. ${ }^{16}$ Chojalactone (3), which has a $\gamma$-butyrolactone structure, was isolated from the combined-culture broth of Streptomyces sp. CJ-5 and T. pulmonis. Chojalactone exerts anticancer activity against P388 murine leukemia cells. Niizalactam (4) was found in the broth of combined-culture with Streptomyces sp. NZ-6 and T. pulmonis. ${ }^{17}$ 5-Alkyl-1,2,3,4-tetrahydroquinolines (5-12), having a tetrahydroquinone skeleton, were isolated from the combined-culture broth of Streptomyces nigrescens HEK616 and T. pulmonis. 5-Alkyl-1,2,3,4tetrahydroquinolines $s$ exhibit antifungal activity against yeasts. ${ }^{18,19}$ 


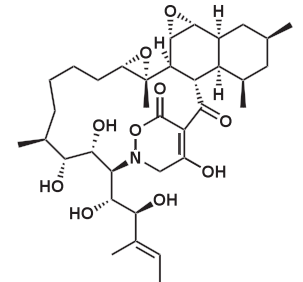

Alchivemycin A (1)

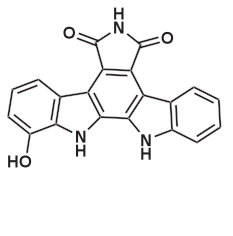

arcyriaflavin E (2)

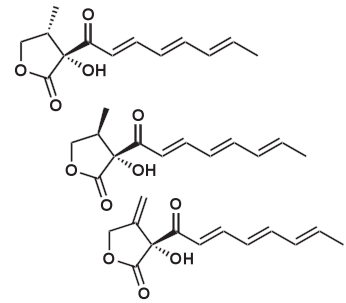

chojalactones (3)

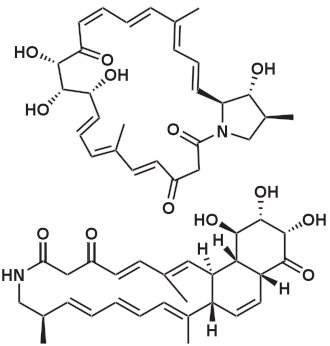

niizalactams (4)

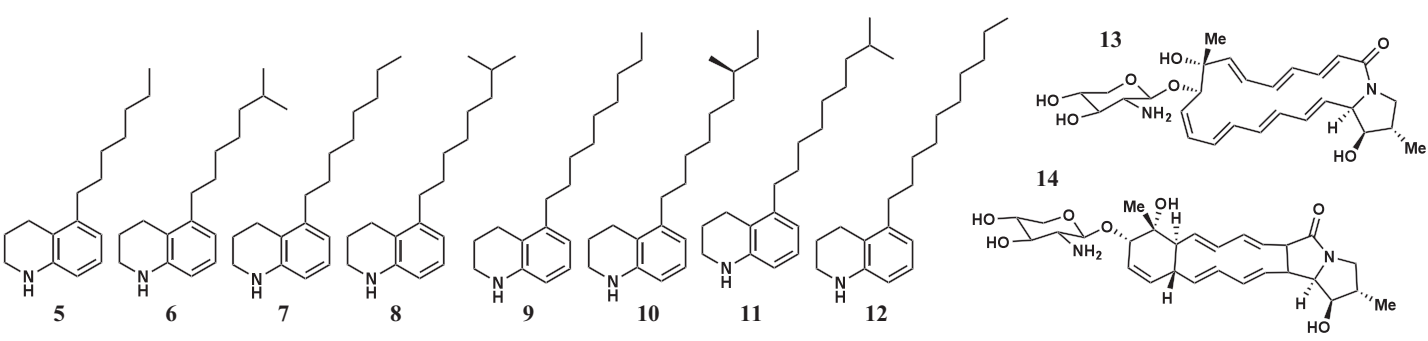

5-Alkyl-1,2,3,4-tetrahydroquinolines (5aTHQ) (5-12)

Ciromicins $(\mathbf{1 3}, \mathbf{1 4})$

Figure 5 Novel secondary metabolites isolated by combined-culture. Alchivemycin A (1), arcyriaflavin E (2), chojalactone (3), niizalactam (4), 5-alkyl-1,2,3, 4-tetrahydroquinolines (5aTHQs) (5-12), ciromicins (13 and 14).

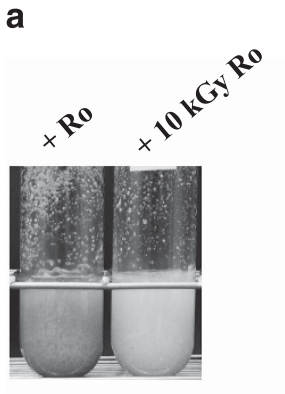

$\mathbf{b}_{+ \text {R. opacus }}$

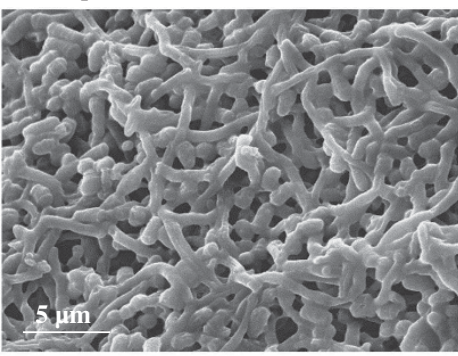

$+T$. pulmonis
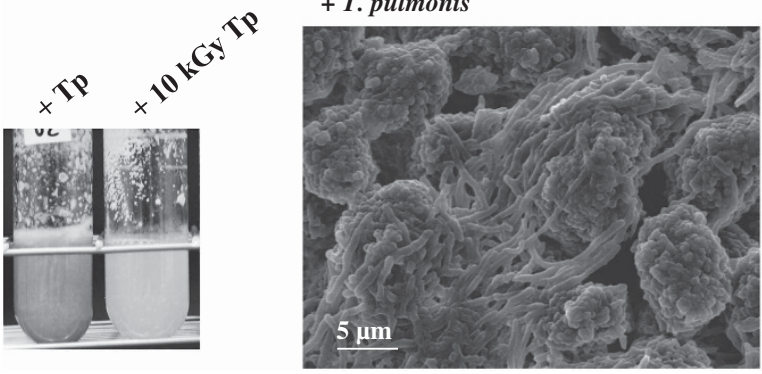

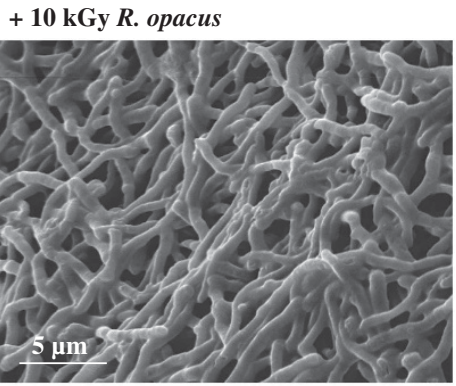

+ 10 kGy T. pulmonis

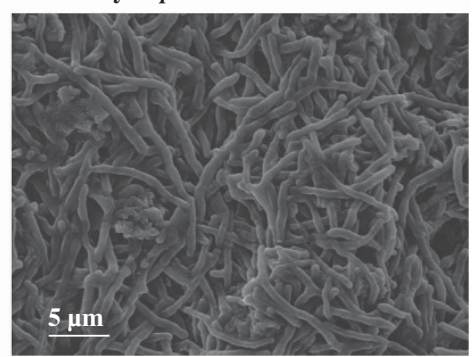

Figure 6 Liquid culture and electron micrographs of combined-culture compared with irradiated dead cells and live cells. (a) Liquid culture on A-3M medium. +Ro: combined-culture with R. erythropolis and S. lividans. +10 kGy Ro: combined-culture with dead Rhodococcus opacus irradiated with 10 kGy and S. lividans. +Tp: combined-culture with T. pulmonis and S. lividans. $+10 \mathrm{kGy} \mathrm{Tp}$ : combined-culture with dead T. pulmonis irradiated with $10 \mathrm{kGy}$ and S. lividans. (b) Electron micrographs of combined-culture. The filamentous structure is Streptomyces mycelium in four all micrographs. Rod-shaped (+Ro) and clamp (+Tp) structures are R. opacus and T. pulmonis, respectively. A full colour version of this figure is available at the Journal of Antibiotics journal online.

Moreover, inspired by our combined-culture method, Bachmann and colleagues ${ }^{20}$ identified ciromicins (13 and 14) in a combinedculture of the MACB Rhodococcus wratislaviensis and the actinomycete Nocardiopsis sp. FU 40. In addition, combined-culture was applied to increase production. We demonstrated that the heterologous production of goadsporin, stauroposporine and rebeccamycin in S. lividans increased when combined-cultures were performed with T. pulmonis, Rhodococcus erythropolis and Corynebacterium glutamicum. $^{21}$
Do actinomycetes have a recognition mechanism for others?

Scanning electron microscopic analysis of combined-cultures with $\mathrm{MACB}$ and actinomycetes showed that MACB adhered closely to actinomycetes. The resulting images suggested that actinomycetes recognized the adhesion of other microorganisms and altered their secondary metabolism. It was also considered that the mycolic acid layer on the outer side is an important factor for adhering. To verify this hypothesis, dead MACB possessing intact mycolic acid assembled on the outer membrane were prepared and cultured in 
combined-culture with $S$. lividans, to examine red pigment production-inducing ability. ${ }^{22}$ Surprisingly, when the dead cells were cultured with S. lividans, red pigment production was not observed (Figure 6a). Electron micrographs at the time of combined-culture with living bacteria and killed bacteria showed that the viable MACB were entwined tightly with mycelia of S. lividans, whereas the dead cells could not entwine (Figure $6 \mathrm{~b}$ ). ${ }^{22}$ These results suggested that the direct interaction with MACB and the sustained contact stimulation of the mycelia of actinomycetes may cause Streptomyces to alter their specialized metabolism in combined-culture.

\section{CONCLUDING REMARKS}

In this review, I described secondary metabolite production using microbial interactions in combined-culture. Co-culture is a simple method in which two different microorganisms are cultured together; scale up can easily be achieved by scale up of the inoculation of MACB and more chemical reagent if needed. The most striking feature in combined-culture is that both strains grow depending on the culture scale; thus, preparation of large amounts of MACB is easier than preparation of chemical activator compounds, giving this method advantages in industrialization. Although the concept of applying co-culture for secondary metabolite production is not new, ${ }^{23}$ the bacterial combinations that are effective for secondary metabolism activation have not been determined. It is expected that combinedculture with MACB and actinomycetes may be useful for stimulation of secondary metabolism in a variety of actinomycetes. Moreover, there are no relationships among the structural classes of compounds that have already been discovered by combined-culture. This feature of the combined-culture is important for the exploration of new natural compounds.

Actinomycetes are soil microorganisms that coexist with various organisms, such as plants, protozoa and insects living in the soil environment. A series of studies on the combined-culture revealed that actinomycetes can show changes in secondary metabolism following contact with other living things, suggesting that actinomycetes adapt to the natural environment and can alter their secondary metabolic patterns as needed. However, it is still unclear why actinomycetes produce many types of antibiotics. Although these antibiotics may be advantageous for survival and coexistence with other living organisms, it is difficult to explain the energy input into such abundant secondary metabolic processes. In addition, combinedculture is associated with activation of secondary metabolism by physical contact and provides a mechanism through which microorganisms can recognize other organisms in the outer world. Further studies on combined-culture may provide answers to these interesting questions.

\section{CONFLICT OF INTEREST}

The author declares no conflict of interest.

\section{ACKNOWLEDGEMENTS}

The research presented herein was carried out in the Laboratory of Microbial Metabolic Potential, The University of Tokyo. I thank the past and present members of my laboratory and the collaborators who have carried out a substantial amount of the work described herein. In particular, I thank Dr Shumpei Asamizu, Dr Taro Ozaki, Dr Yoshinori Sugai, Professor Yasuo Ohnishi, Professor Ikuro Abe, Professor Hiroaki Suga,
Professor Teruhiko Beppu and Professor Sueharu Horinouchi (The University of Tokyo), Ms Yukiko Mori and Ms Mizuho Nakaho (Toyama Prefectural University), and Professor Hideaki Kakeya (Kyoto University). The research presented herein was supported in part by a Grant-in-Aid from IFO, Institute for Fermentation, Osaka, JSPS KAKENHI (grant number 25108707) and JSPS KAKENHI for Scientific Research on Innovative Areas (grant number JP16H06444).

1 Cragg, G. M. \& Newman, D. J. Natural products: a continuing source of novel drug leads. Biochim. Biophys. Acta. 1830, 3670-3695 (2013).

2 Nett, M., Ikeda, H. \& Moore, B. S. Genomic basis for natural product biosynthetic diversity in the actinomycetes. Nat. Prod. Rep. 26, 1362-1384 (2009).

3 Scherlach, K. \& Hertweck, C. Triggering cryptic natural product biosynthesis in microorganisms. Org. Biomol. Chem. 7, 1753-1760 (2009).

4 Zarins-Tutt, J. S. et al. Prospecting for new bacterial metabolites: a glossary of approaches for inducing, activating and upregulating the biosynthesis of bacterial cryptic or silent natural products. Nat. Prod. Rep. 33, 54-72 (2016).

5 Ochi, K. Insights into microbial cryptic gene activation and strain improvement: principle, application and technical aspects. J. Antibiot. (Tokyo) 70, 25-40 (2017).

6 Horinouchi, S. \& Beppu, T. Hormonal control by A-factor of morphological development and secondary metabolism in Streptomyces. Proc. Jpn Acad. Ser. B Phys. Biol. Sci. 83, 277-295 (2007)

7 Onaka, H., Tabata, H., Igarashi, Y., Sato, Y. \& Furumai, T. Goadsporin a chemical substance which promotes secondary metabolism and morphogenesis in streptomycetes. I. Purification and characterization. J. Antibiot. (Tokyo) 54, 1036-1044 (2001)

8 Igarashi, Y. et al. Goadsporin, a chemical substance which promotes secondary metabolism and morphogenesis in streptomycetes. II. Structure determination. J. Antibiot. (Tokyo) 54, 1045-1053 (2001).

9 Onaka, H., Nakaho, M., Hayashi, K., Igarashi, Y. \& Furumai, T. Cloning and characterization of the goadsporin biosynthetic gene cluster from Streptomyces sp. TP-A0584. Microbiology 151(Pt 12), 3923-3933 (2005)

10 Ozaki, T. et al. Insights into the biosynthesis of dehydroalanines in goadsporin. Chembiochem 17, 218-223 (2016).

11 Ozaki, T. et al. Dissection of goadsporin biosynthesis by in vitro reconstitution leading to designer analogs expressed in vivo. Nat. Commun. 8, 14207 (2017).

12 Haginaka, K. et al. Genetic approaches to generate hyper-producing strains of goadsporin: the relationships between productivity and gene duplication in secondary metabolite biosynthesis. Biosci. Biotechnol. Biochem. 78, 394-399 (2014).

13 Onaka, H., Mori, Y., Igarashi, Y. \& Furumai, T. Mycolic acid-containing bacteria induce natural-product biosynthesis in Streptomyces species. Appl. Environ. Microbiol. 77, 400-406 (2011).

14 Igarashi, Y. et al. Alchivemycin A, a bioactive polycyclic polyketide with an unprecedented skeleton from Streptomyces sp. Org. Lett. 12, 3402-3405 (2010).

$15 \mathrm{Kim}$, Y., In, Y., Ishida, T., Onaka, H. \& Igarashi, Y. Biosynthetic origin of alchivemycin A, a new polyketide from Streptomyces and absolute configuration of alchivemycin B. Org. Lett. 15, 3514-3517 (2013).

16 Hoshino, S. et al. Arcyriaflavin E, a new cytotoxic indolocarbazole alkaloid isolated by combined-culture of mycolic acid-containing bacteria and Streptomyces cinnamoneus NBRC 13823. J. Antibiot. (Tokyo) 68, 342-344 (2015).

17 Hoshino, S., Wakimoto, T., Onaka, H., Abe, I. \& Chojalactones, A.-C. cytotoxic butanolides isolated from Streptomyces sp. cultivated with mycolic acid containing bacterium. Org. Lett. 17, 1501-1504 (2015).

18 Sugiyama, R. et al. 5-Alkyl-1,2,3,4-tetrahydroquinolines, new membrane-interacting lipophilic metabolites produced by combined culture of Streptomyces nigrescens and Tsukamurella pulmonis. Org. Lett. 17, 1918-1921 (2015).

19 Sugiyama, R. et al. Discovery and total synthesis of streptoaminals: antimicrobial $[5,5]$-spirohemiaminals from the combined-culture of Streptomyces nigrescens and Tsukamurella pulmonis. Angew. Chem. Int. Ed. Engl. 55, 10278-10282 (2016).

20 Derewacz, D. K., Covington, B. C., McLean, J. A. \& Bachmann, B. O. Mapping microbial response metabolomes for induced natural product discovery. ACS Chem. Biol. 10, 1998-2006 (2015).

21 Onaka, H. et al. Mycolic acid-containing bacteria activate heterologous secondary metabolite expression in Streptomyces lividans. J. Antibiot. (Tokyo) 68, 594-597 (2015).

22 Asamizu, S., Ozaki, T., Teramoto, K., Satoh, K. \& Onaka, H. Killing of mycolic acidcontaining bacteria aborted induction of antibiotic production by streptomyces in combined-culture. PLOS ONE 10, e0142372 (2015).

23 Bertrand, S. et al. Metabolite induction via microorganism co-culture: a potential way to enhance chemical diversity for drug discovery. Biotechnol Adv. 32, 1180-1204 (2014). 